\section{CONDUCIRY EXPLORAR: EL SABOR DE SUMERGIRSE EN EL ESPACIO ARQUITECTÓNICO}

\begin{abstract}
Luiz Amorim
Arquitecto y urbanista, formado por la Universidade Federal de Pernambuco, en 1982, donde es profesor asociado. Completó su doctorado en la University College London, en 1999. Revista EN BLANCO. Nº 9. Arquitectura Brasileña. Valencia. Año 2012. (Páginas 122-123) ISSN 1888-5616. Recepción: 10_11_2011. Aceptación: 13_01_2012.
\end{abstract}

Palabras clave: Construcción del espacio, comportamiento, movimiento, observación, exposición.

Resumen: Nos interesa observar en qué medida los elementos estructurales pueden ser vistos como protagonistas en la constitución del espacio, sea como conductor del movimiento y modelador de la vista, o como organizador de ambientes para la ocupación humana. Por tanto, como medio para estructurar la interacción entre ocupantes del espacio de la arquitectura. La demostración de esa condición propia de la arquitectura puede ser realizada a través de algunas de las obras aquí reunidas, particularmente las de naturaleza pública: la plaza Turca, el Centro Burle Marx y la Galeria Varejão.

Keywords: Construction of space, behavior, movement, observation, exhibition. Abstract: We want to observe to what extent the structural elements can be seen as leading constituents of space, be it as a driver of the movement, or shaper of the eye, or as organizer of environments for human occupation. Hence, as a mean to structure the interaction among the inhabitants of the architectural space. A demonstration of this properly architectural condition can be performed by analyzing some of the works gathered here, especially the ones with a public nature: the Turkish Square, the Burle Marx Center and the Varejão Gallery.

En el oficio de albergar las diversas manifestaciones de la naturaleza humana, los edificios son moldeados según presupuestos que privilegian la emergencia de algunas de esas manifestaciones en detrimento de otras. Son esas prioridades las que distinguen, en cierta medida, los tipos de edificaciones. La residencia que habitamos, el estudio en el que trabajamos, el templo donde sublimamos las limitaciones del ser humano, son ambientes que privilegian ciertas condiciones de ocupación y de movimiento en el espacio y que proporcionan distintas modalidades de interacción entre personas.

La formalidad ritualística del templo religioso, por ejemplo, se obtiene por la constitución de un cuerpo íntegro en el cual estructura, forma, epidermis y espacio son orquestados para obtener el manifiesto sentido de la superación metafísica. En eventos de alta complejidad, como en festividades de devoción a los entes superiores, en ceremonias de nupcias o entronización, la arquitectura del edificio se manifiesta como copartícipe, y no como mero escenario del evento. De hecho, la edificación constituye los medios para su realización, pero también las oportunidades para la ocurrencia de eventos no previstos. El cine ha explorado sistemáticamente lo inesperado en ceremonias de nupcias - ver el clásico The Graduate (1967), de Mike Nichols, por ejemplo, o en rituales de sociedades tribales o civilizaciones "perdidas" - ver los clichés hollywoodianos en películas de aventuras.

Es cierto que las obras cinematográficas, como piezas de ficción, están circunstanciadas a la interpretación de sus autores que resaltan ciertos aspectos en detrimentos de otros, como forma de constituir una narrativa coherente. Raras son las obras que consiguen retratar con fidelidad la multiplicidad de eventos abrigados por la obra arquitectónica u ofrecer medios para su comprensión. Mon Oncle (1958) y Playtime (1967), de Jacques Tati permiten, por medio de la discusión del conflicto entre las distintas culturas, subculturas urbanas y modos de vida, observar sus efectos sobre las formas de interfaz mediadas por la arquitectura, sean en el ambiente doméstico, laboral o de la ciudad.

El argumento esgrimido no es nuevo. De hecho, la relación entre arquitectura y comportamiento ha sido estudiada extensamente desde los años 60, abarcando concepciones casi deterministas -si la arquitectura presenta tales propiedades, el comportamiento humano se dará de determinada manera, hasta aquellas de naturaleza probabilística- si la arquitectura presenta tales propiedades, es probable que ciertas condiciones de ocupación y movimiento se aproximen a patrones observados in situ.

Pero, ¿Cual es la relevancia de esa cuestión para el contexto específico del conjunto de obras aquí reunido?

Fundamentalmente, para observar en qué medida los elementos estructurales pueden ser vistos como protagonistas en la constitución del espacio, sea como conductor del movimiento y modelador de la vista, o como organizador de ambientes para la ocupación humana. Por tanto, como medio para estructurar la interacción entre ocupantes del espacio de la arquitectura. La demostración de esa condición propia de la arquitectura puede ser realizada a través de algunas de las obras aquí reunidas, particularmente las de naturaleza pública: la plaza Turca, el Centro Burle Marx, y la Galeria Varejão. Hay en las obras referidas una intrínseca relación entre los componentes estructurales - menos evidentes en la Plaza Turca -, y la apreciación de obras de arte y del paisaje urbano y natural. El movimiento de personas, sea fruto de la conducción forzada o de la libre exploración del espacio arquitectónico, también está estructurado según una relación dinámica entre observación y exposición (ver y ser visto).

En las dos edificaciones que forman parte del Instituto Cultural Inhotim, los respectivos forjados de cubierta son accesibles y se prestan como inicio o fin de un recorrido (Galeria Varejão) o de innumerables recorridos exploratorios (Centro Burle Marx), o como lugares de permanencia, incluso teniendo en cuenta la exposición de los elementos de la naturaleza. En la Galeria Varejão, el interés reside en preparar y conducir al visitante observador del arte del majestuoso ambiente natural hacia el controlado y aislado interior. El rigor de la conducción del movimiento y de la visual es construido antes de adentrarse en la galería, sea en la disposición del acceso por la pasarela en el eje de simetría del volumen macizo de hormigón, en el pavimento superior, o sea, de distinta manera, en el desplazamiento del acceso en el pavimento inferior de la simetría referida.

En la plataforma superior, el plano de disfrute del paisaje y de la obra Passarinhos - de Inhotim a Demini (2003-2008) se articula con la gran nave 
de exposición del primer piso por la rampa enclaustrada que la circunda, en el primer gesto de torsión de la secuencia de movimiento concebida por Rodrigo Cerviño Lopes. Simétricamente dispuesta en la planta baja, la misma torsión es orquestada a lo largo de las paredes expositivas. La escalera de acceso al pavimento superior define el eje de simetría entre los movimientos de torsión periféricamente distribuidos en la planta baja y en la planta superior, y es la responsable de crear el movimiento giratorio de apreciación de la bellísima Celacanto Provoca Maremoto (2004-2008). Envuelto por la obra, inmerso en el espacio de simétrica inducción al movimiento, la ruta centrífuga lleva siempre a movimientos periféricos hacia la parte superior o para abajo, de acuerdo con la ruta o el deseo del visitante. En ese juego, los elementos estructurales son lanzados de forma que permite que espacio y materia conduzcan la experiencia arquitectónica, sin grandes oportunidades para la urgencia de eventos no previstos.

En el Centro Burle Marx, de Brasil y Zasnicoff, edificio que tiene como principal atribución albergar actividades de carácter educacional, los ejes de circulación son variados y superpuestos. Variados, puesto que están dirigidos hacia espacios específicos, como el auditorio, sala de ocio, biblioteca, café, o utilizan la estructura edificada para superar barreras -el lago y la topografía, o convertir el camino en más placentero- con sombras, con vistas sobre el paisaje, con la proximidad al agua, etc. Superpuesto, debido al forjado de cubierta, que también es lugar para ver y ser visto, es la plataforma la que permite el transporte de niveles, según el sistema de escaleras y rampas. Hay en el Centro un fuerte sentido direccional del movimiento hacia (edificio como destino) y a través (edificio como pasajel y de la generación de amplios espacios de permanencia, cuyo foco de atención reside en propiciar la interacción entre los diversos visitantes de Inhotim. El propósito es alcanzado por medio del lanzamiento de un sistema modulado de columnas de hormigón, soporte del forjado de cubierta, y de la adaptación de la edificación a la topografía existente. La única nota disonante en esta feliz disposición está en la plataforma de apreciación de la obra Narcissus Garden Inhotim (2009), de Yayoi Kusama, que no permite la transposición hacia el margen opuesto del lago, forzando el retorno de los visitantes por el mismo recorrido: una consonancia indeseable con el baile enclaustrado de las esferas de Kusama.

A diferencia de los espacios sujetos al control privado, como en Inhotim, la ciudad se presenta para la libre ocupación y movimiento, según las condiciones impuestas por las normas sociales y por los hábitos culturales. En la Plaza Turca, de Alban e Gramacho, el ejercicio que se aborda es volver apacible un trecho urbano de la árida ciudad de Juazeiro.
La estructuración del espacio público se realiza según la disposición modular de columnas soportan superficies entramadas para crear espacios de sombra. A la malla creada son adicionados bancos que, según su disposición, estructuran el sistema de recorridos, configurando la propiedad más importante de las plazas bien configuradas: la articulación entre líneas de movimiento y áreas de permanencia. Nuevamente, ver y ser visto son actos potenciados como mecanismos necesarios para la interacción social, donde lo imprebisible manda.

No seria indebida la inserción de una cuarta obra para ejemplificar una de las mas felices realizaciones en la cual los componentes estructurales son suficientes para establecer distinciones espaciales. La Capilla de Nuestra Señora de la Concepción, de Paulo Mendes da Rocha y Eduardo Colonelli (2004-2006), construida en Recife, parte de una edificación arruinada que se presentaba para su intervención como un rectángulo definido por paredes de mampostería mixta rodeados por arcos también de mampostería, parcialmente completada. Al transformarla en un templo religioso, los arquitectos se adaptaron a las demandas que los eventos litúrgicos católicos que se prescriben y son delimitados por el conjunto de ambientes que lo caracterizan - el nártex, la nave, el altar, el coro, la sacristía y el campanario.

Lo que tiene de particular la Capilla de Nuestra Señora de la Concepción son los medios utilizados para concebir la espacialidad del templo católico, o sea, la disposición de las dos columnas de soporte de la losa de cubierta. Su disposición en el espacio rectangular de la ruina preexistente es suficiente para estructurar la experiencia ritualística, según la disposición secuencial y dual entre el nártex y el altar, de acuerdo con las reglas seculares. La columna-altar es foco, depositaria de la hermosa imagen votiva, de Francisco Brennand. La "columna-biombo" es barrera al movimiento lineal y establece el desvío en el recorrido para encuadrarlo, ahora en el interior. y dividir la nave en dos alas. La misma columna, ahora columna-coro, reduce la altura libre interior al adentrarse en la capilla. Ningún otro elemento arquitectónico es necesario para constituir el espacio religioso -jerarquía, oposición, movimiento, ocupación, apreciación, sublimación, todos manifiestos según la disposición de los elementos estructurales en el espacio. A pesar de la forma inusitada, el pequeño templo no difiere de aquellos encontrados desde los primeros siglos de ocupación del territorio brasileño.

En el diálogo propuesto entre los elementos estructurales y el espacio arquitectónico de la Capilla de Nuestra Señora de la Concepción se forjan ricamente la superposición de guiones formales secularmente constituidos y una pléyade de eventos no previstos, propias para la construcción de numerosas narrativas ficticias -o no.
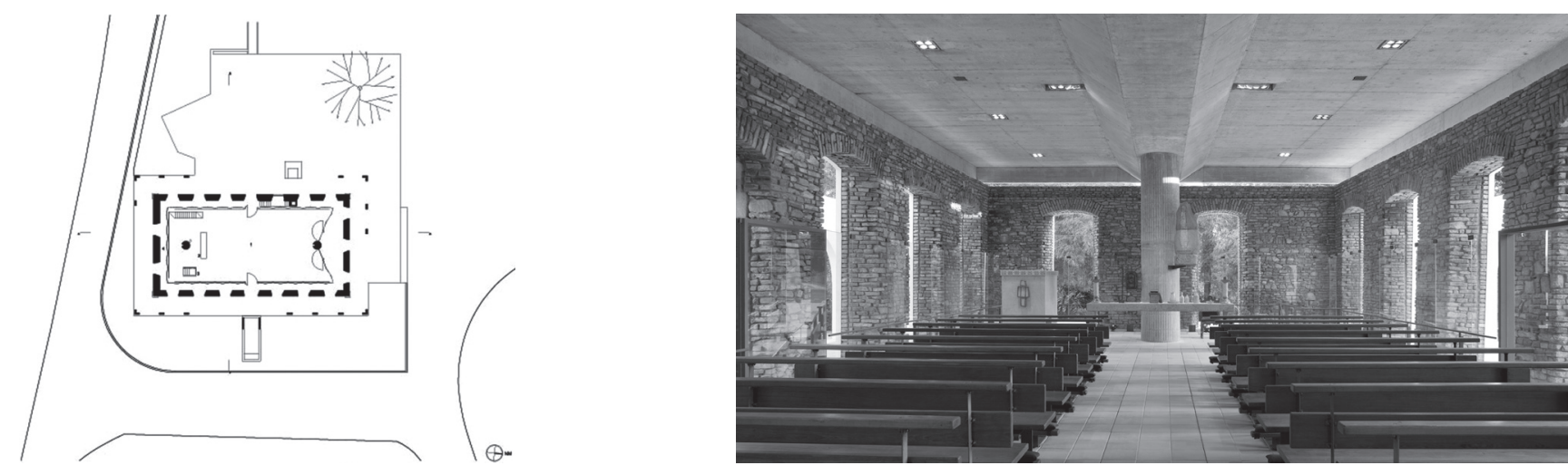